\title{
The Chemical Reaction Optimization Approach to Solving the Environmentally Sustainable Network Design Problem
}

\author{
W. Y. Szeto*, Yi Wang, S. C. Wong \\ Department of Civil Engineering, The University of Hong Kong, Hong Kong SAR, China
}

\begin{abstract}
Nowadays, the decision makers in the transportation industry are being urged to instill environmental costs into road network design decision-making because road traffic affects the environment and health. The design of a road network should be not only cost-effective but also environmentally sustainable. This article proposes a new network design problem (NDP) that takes both vehicle emissions and noise into account. This proposed environmentally sustainable NDP is formulated as a discrete bi-level program. The lower level problem is formulated as user-equilibrium assignment. The upper level problem determines the optimal road capacity expansion to minimize the total costs of emissions, noise, and travel time with the considerations of budgetary and capacity improvement constraints. The proposed problem is solved by an enhanced version of a new metaheuristic named Chemical Reaction Optimization $(C R O)$, and its parameters are tuned by our proposed tuning procedure. Two benchmark road networks with different demand levels are used to evaluate the performance of the enhanced CRO and illustrate the properties of the problem. The results show that there were tradeoffs between emissions, noise, and travel time costs, and that the enhanced CRO outperformed Genetic Algorithm (GA) on more than half of the testing scenarios and had a comparable performance on certain test scenarios compared with GA.
\end{abstract}

\section{INTRODUCTION}

The bi-level Network Design Problem (NDP) is to find the optimal decision on selecting either link improvements or link additions to an existing road network in the upper level problem, while accounting for the route choice behavior of network users in the lower level problem (Yang et al., 1998). This problem has been receiving attention, because with the economic development, the demand for travel on roads is growing at a rate faster than the urban transportation system can accommodate but the budget and land for expanding the system are limited. Sometimes, this problem has been generalized to consider toll setting (e.g., Shepherd, 2012; Li et al., 2012), signal control (e.g., Hawas, 2011), ramp metering, and other transportation network design problems.

The objective of the upper level problem is to optimize a given system performance measure, and is traditionally to minimize the total system travel time or cost (e.g., $\mathrm{Ng}$ and Waller, 2009a, b; Long et al., 2010). Some studies also include the investment costs into the objective function (e.g., Meng et al., 2001; Xu et al., 2009; Wang and Lo, 2010). Others consider reserve capacity (e.g., Chen et al., 2006), consumer surplus (e.g., Szeto and Lo, 2008; Lo and Szeto, 2009), equity (e.g., Szeto and Lo, 2006; Duthie and Waller, 2008) and change in social surplus (e.g., Szeto et al., 2010), and profit (e.g., Shepherd, 2013) in the objective function. However, road traffic also imposes a profound influence on the environment. To have a sustainable transportation development, it is necessary to incorporate the effects of road traffic on the environment and consider environmental sustainability in transportation network design. This leads to a new research area of the bi-level NDP, namely the bi-level transportation network design problem with environmental considerations (BTPE), or equivalently the environmentally sustainable NDP, which considers the negative effects of road traffic on the environment and has been recently reviewed by Szeto et al. (2012). The model structure of BTPE is similar to that of a typical bi-level NDP, but the upper level problem includes environmental impact measures such as noise or vehicle emissions in either the objective function or constraints (e.g., Huang et al., 2009; Li et al., 2012).

To capture the environmental consideration in the objective function, the environmental objective is sometimes treated as the only objective in the model (e.g., Ferguson et al., 2010), but very often is combined with other objectives as one objective function using the weighted sum approach (e.g., Kim and Kim, 2006; Qiu and Chen, 2007; Jia et al., 2009), or is one of the objectives in the multi-criteria 
optimization model (e.g., Ferguson et al., 2012; Chen and Xu, 2012). In the first approach, Ferguson et al. (2010) solved the emissions network design problem to minimize the total network emissions including three air pollutants: hydrocarbons $(\mathrm{HC})$, nitrogen oxides $\left(\mathrm{NO}_{\mathrm{x}}\right)$, and carbon monoxide (CO). They are considered because they are harmful to human health and/or cause climate change - $\mathrm{CO}$ increases the chance of people having cardiovascular diseases and impede the psychomotor functions; HC form ground-level ozone and the smog to cause deleterious health and greenhouse effects; $\mathrm{NO}_{\mathrm{x}}$ form ground-level ozone to create nitric acid causing serious respiratory problems.

In the existing studies using the weighted sum approach, various objectives are considered and combined into one single objective. For example, Kim and Kim (2006) incorporated the emission cost into the objective function as the sum of social costs including network travel time cost, maintenance cost, accident cost, and vehicle operation cost. Qiu and Chen (2007) included the environmental pollution cost in the objective function together with the network travel time cost, investment cost, land use and energy consumption costs. Jia et al. (2009) proposed cumulative costs including emission cost over the modeling horizon.

In the third approach, more than one objective is also considered simultaneously to determine the Pareto frontier. In the literature, only various objectives are considered. For instance, Wismans et al. (2011) considered the total emissions of $\mathrm{CO}_{2}$, noise, and total travel time in their multi-objective optimization model. Ferguson et al. (2012) explored the tradeoffs between designing a road network for minimal congestion and designing it for minimal vehicle emissions. Chen and $\mathrm{Xu}$ (2012) considered total CO emissions, total travel time, and the maximum ratio of origin-destination travel times after and before capacity enhancement in their multi-objective optimization model.

As shown in the above, few researchers incorporated both noise and vehicle emission costs into BTPE simultaneously. To fill this research gap in the network design area, we propose a more comprehensive bi-level model for incorporating the costs of noise and different types of vehicle emissions into BTPE. The upper level minimizes the sum of total system travel time cost, emission cost and noise cost. Three key pollutants are chosen for emission cost calculation: $\mathrm{CO}, \mathrm{HC}$ (also known as volatile organic compounds (VOC)) and $\mathrm{NO}_{\mathrm{x}}$. They are chosen because of their respective and combined significant impacts on human health and the environment (Ferguson et al., 2012). The noise cost is calculated by the model proposed by Delucchi and Hsu (1996). The decision is to determine the optimal selection of link improvements in road networks under limited budget and capacity improvement constraints. The capacity improvement is modeled by a discrete decision variable so our problem belongs to the bi-level discrete NDP.

Since our bi-level discrete NDP is nonlinear, nonconvex and NP-hard, it is difficult to solve for global optima exactly and efficiently, especially for large network applications. Hence, the recent trend is to develop, apply and improve meta-heuristics which can obtain good solutions efficiently. The following metaheuristics are some examples that have been used for solving NDP and BTPE:

- genetic algorithm (GA) (e.g., Ng et al., 2009; Chen et al., 2010; Sharma and Mathew, 2011; Szeto and Wu, 2011),

- simulated annealing (SA) (e.g., Meng and Yang, 2002),

- particle swarm optimization (e.g., Zhang and Gao, 2007; Miandoabchi et al., 2012a),

- artificial bee colony (ABC) (e.g., Szeto et al., 2011), and

- clonal selection algorithm (e.g., Miandoabchi et al., 2012b).

The performances of some of the above heuristics were compared between each other. However, which metaheuristic is better depends on the problem and the demand setting considered. For example, Karoonsoontawong and Waller (2006) found that GA outperforms SA and random search in solving their linear, continuous network design problem, but Jia et al. (2009) found that SA performs better than GA and $\mathrm{ABC}$ in solving their problem. Xu et al. (2009) investigated the performance of GA and SA under different demand conditions. They found that SA was more efficient than GA in solving the nonlinear continuous network design problem, and much more computational effort was needed for GA to achieve the same optimal solution as SA. However, when demand was light, GA could reach a more optimal solution at the expense of more computation time.

The above meta-heuristics are not the only meta-heuristics that can be used to solve NDP and BTPE. Indeed, many meta-heuristics exist but not all of them have been applied to solve these problems. One of such example is chemical reaction optimization (CRO), which has been recently proposed by Lam and Li (2010). It mimics the interactions of molecules in a chemical reaction to reach a low energy stable state. It is a variable population-based metaheuristic, where the total number of solutions kept simultaneously by the algorithm may change from time to time (Lam and $\mathrm{Li}, 2010$ ). CRO has the ability to avoid getting stuck at local minima. Different from other heuristics, CRO allows the diversification and intensification of solutions to occur automatically, rather than using a fixed sequence of operators for these purposes. Moreover, as Lam and $\mathrm{Li}$ (2010) indicated, CRO may be considered an optimization algorithm which allows the users to use their favorable heuristic components for specific optimization problems, owing to the changeable components including criteria and mechanisms of various operators. The extents of intensification and diversification on solution searching are controlled easily by operators. Hence, this meta-heuristic can be applied to a wide range of optimization problems and has already been proven to be well performed in solving classic NP-hard problems, such as the quadratic assignment problem, the resource-constrained project scheduling problem and the channel assignment problem (e.g., Lam and $\mathrm{Li}, 2010)$. It also 
had a wide application in various fields, such as the fuzzy rule learning problem (e.g., Lam et al., 2012), sensor deployment for air pollution monitoring (e.g., Yu et al., 2012) and stock portfolio selection (e.g., Xu et al., 2011). However, the performance of $\mathrm{CRO}$ in solving bilevel problems, including BTPE, has not been known yet. Therefore, we are interested in improving $\mathrm{CRO}$ and testing the performance of the improved algorithm in solving our proposed bilevel problem. Numerical examples are set up to illustrate the performance of the improved CRO, and compare it with GA because GA is the most classical and frequently used metaheuristic, and GA is found to be used widely for solving BTPE (Szeto et al., 2012).

Like other metaheuristics, CRO has parameters that need to be tuned to achieve better results for each problem. However, every problem has its own characteristics and it is time-consuming to determine a good parameter combination for each problem. This study proposes a new method, called the normalized parameter tuning method. This method is to tune the normalized coefficients for certain parameters which are closely related to the objective function value of a category of similar problems. It can help find the suitable parameter combination quicker.

The main contributions of this study lie in the following aspects: firstly, it incorporates the environmental considerations including noise and multiple types of emissions simultaneously into the objective of NDP, in order to illustrate the total environmental cost can be large and should not be ignored; secondly, it improves CRO to solve BTPE and illustrates the effectiveness and potential applications for large transportation networks. Thirdly, it demonstrates the trade-offs between congestion and different environmental objectives. Fourthly, a new parameter tuning method is proposed for CRO implementation to help finding suitable parameter values quicker.

The rest of this article is outlined as follows. Section 2 formulates the problem. Section 3 introduces CRO and the enhancement. Section 4 presents the numerical studies on two benchmark transportation networks and finally, Section 5 gives the conclusion.

\section{PROBLEM FORMULATION}

The proposed problem is formulated as a bi-level problem. The lower level problem captures the behavior of transportation network users while the upper level problem determines the optimal link capacity expansion decision made by the transportation planner. The mathematical details of lower level and upper level problems will be given in Sections 2.1 and 2.2, respectively.

\subsection{Lower level problem}

In this problem, the classic deterministic user equilibrium (UE) principle is adopted to model the travelers' behavioral reaction to transportation planner's link expansion decisions and the demand is assumed to be fixed. Let $A$ be the set of links of the network, $R_{w}$ be the set of routes between origin-destination (O-D) pair $w \in W$, and $W$ be the set of O-D pairs. The travel time on link $a$ is denoted by $t_{a}\left(v_{a}, u_{a}\right)$, $a \in A$ with $v_{a}$ being the flow on link $a$ and $u_{a}$ being the capacity enhancement of link $a$. Then, the lower level problem can be formulated as:

$$
\min _{\mathbf{v}} \sum_{a \in A} \int_{0}^{v_{a}} t_{a}\left(x, u_{a}\right) d x
$$

subject to

$$
\begin{gathered}
\sum_{r \in R_{w}} f_{r}=d_{w}, \forall w \in W \\
f_{r} \geq 0, \forall r \in R_{w}, w \in W
\end{gathered}
$$

where $f_{r}$ is the flow on route $r \in R_{w}, w \in W, \mathbf{v}$ is the vector of link flows and $d_{w}$ is the demand between O-D pair $w \in W$. Here, the link flow $v_{a}$ is defined by

$$
v_{a}=\sum_{r \in R} f_{r} \delta_{a r}, \forall a \in A
$$

where $\delta_{a r}=1$ if route $r$ uses link $a$; and $\delta_{a r}=0$ otherwise.

\subsection{Upper level problem}

2.2.1 Objective. The upper level problem aims at determining which links to be improved in order to minimize the total cost $(T C)$. Other than the traditional total system travel time cost (TSTC), total emission cost (TEC) and total noise cost (TNC) are included simultaneously in the objective function. Mathematically, the objective of the upper level problem can be formulated as:

$$
\min _{\left[u_{a}\right]} T C=T S T C+T E C+T N C
$$

Total system travel time cost

The total system travel time cost is defined by the product of total system travel time and the value of time (VOT).

$$
T S T C=V O T \sum_{a \in A} t_{a} v_{a}
$$

Total emission cost

The total emission cost is defined by summing up the product of the emission cost $E_{a}$ on each link and the flow on that link $v_{a}$ (veh/hour):

$$
T E C=\sum_{a \in A} E_{a} v_{a}
$$

The emission cost $E_{a}$ on link $a$ is given by:

$$
E_{a}=\sum_{k} \phi^{k} Q_{a}^{k}, \forall a \in A
$$

where $\phi^{k}$ is the external cost per unit weight of pollutant $k$, 
and $Q_{a}^{k}$ is the weight of pollutant $k$ emitted on link $a$.

As Penic and Upchurch (1992) pointed out, the weight of different types of pollutants emitted on link $a$ can be expressed a function of link length $l_{a}(\mathrm{ft})$ and link average speed $\bar{S}_{a} \quad\left(\mathrm{ft} / \mathrm{sec}, \bar{S}_{a}=\frac{l_{a}}{t_{a}}\right)$ :

$$
\begin{aligned}
& \text { For CO: } Q_{a}^{C O}=\frac{3.3963 e^{0.014561 \bar{S}_{a}} l_{a}}{1000 \bar{S}_{a}}, \forall a, k \\
& \text { For VOC: } Q_{a}^{V O C}=\frac{2.7843 e^{0.015062 \bar{S}_{a}} l_{a}}{10000 \bar{S}_{a}}, \forall a, k \\
& \text { For } \mathrm{NO}_{\mathrm{x}}: Q_{a}^{N O_{x}}=\frac{1.5718 e^{0.040732 \bar{S}_{a}} l_{a}}{10000 \bar{S}_{a}}, \forall a, k
\end{aligned}
$$

Total noise cost

The total noise cost $T N C$ is calculated by the model proposed by Delucchi and Hsu (1996). According to this model, the external noise cost is equal to the area-noise level $A N_{r, h}$ $\left(\mathrm{dBA}-\mathrm{mi}^{2}\right)$ (defined as the sum of the excess noise levels in the affected area), multiplied by the density of housing units exposed to traffic noise above a threshold $H D$ (units $/ \mathrm{mi}^{2}$ ), the median annualized value of housing units exposed to traffic noise above a threshold $P$ (\$/unit), the percentage of annualized housing value lost for each decibel of noise over the threshold level $H V$ and a scaling factor to account for the noise costs in non-residential areas $\left(\left(T^{\prime}+T\right) / T\right)$, where $T^{\prime}$ is the average amount of time spent away from one's home in places where motor-vehicle noise can be a problem and $T$ is the average amount of time spent in or around one's home.

The model is formulated by:

$$
\begin{aligned}
& T N C=\left(\sum_{r}\left(\sum_{h} A N_{r, h}\right) \cdot H D \cdot P\right) \cdot H V \cdot \frac{T^{\prime}+T}{T} \\
& A N_{r, h}=\frac{l_{r, h}}{5280} \cdot\left(\left(\int_{d_{e}}^{d_{t^{*}}} \operatorname{Leq}(d)_{r, h}\right)-A N B_{r, h}\right), \forall r, h
\end{aligned}
$$

where $l_{r, h}$ (mile) is the total length of road, while subscript $r$ represents different types of roads (such as interstate, other freeway, principal arterial, minor arterial, collector street and local road) and subscript $h$ represents the height-class of noise barriers (such as none, low, medium, and high) along the road. Due to the insufficient information of the road hierarchy and noise barrier used in specific urban road system, in this study, we adopt the parameter values (see Section 4.1) of the base case in Delecchi and Hsu's model for each link $a$ and the road type is selected as "other freeway". $d_{e}$ and $d_{t^{*}}$ are, respectively, the equivalent distance from the roadway to the closest residence $(\mathrm{ft})$ and the equivalent distance from the roadway to the point at which traffic noise drops to the threshold level $t^{*}(\mathrm{ft}) . A N B_{r, h}(\mathrm{dBA}-\mathrm{ft})$ is the sum of the noise-damage threshold $t^{*}$ (which is a function of road type $r$ and noise barrier of height-class $h$,) over the width of the affected area. (i.e., $t^{*}\left(d_{t^{*}-} d_{e}\right)$ ).

The equivalent sound level $\operatorname{Leq}(d)_{a, h}$ is calculated by a submodel:

$$
\begin{aligned}
\operatorname{Leq}(d)_{a, h} & =10 \cdot \log _{10}\left(0.0296 \cdot \frac{\varphi^{\prime}}{180} \cdot v_{a} \cdot K_{a} \cdot\left(\frac{50}{d}\right)^{1+\alpha}\right) \\
& -B_{h}, \forall a, h
\end{aligned}
$$

where $\varphi^{\prime}$ is the equivalent subtending angle, $\alpha$ is the ground-cover coefficient (unitless) and $B_{h}(\mathrm{dBA})$ is the reduction in the noise level due to a barrier with height-class $h . K_{a}$ is the total noise-energy emissions from all types of vehicles. Due to the insufficient information of vehicle types on the road, all vehicles are assumed to be light-duty autos. Mathematically, $K_{a}$ is expressed as:

$$
K_{a}=\frac{1}{\bar{S}_{a} \cdot\left(\bar{S}_{a}^{4.174}+10^{0.115}+10^{C_{a}}\right)}, \forall a \in A
$$

where $\overline{S_{a}}$ is the average speed (miles/hour) of all the vehicles on link $a ; C_{a}$ is the weighted average of exponent for cruising and the exponent for accelerating for the autos on link $a$.

2.2.2 Upper level constraints. The upper level problem also contains the following budget constraint to limit the capacity that can be added to each candidate link:

$$
\sum_{a \in A} \kappa_{a} l_{a} u_{a} \leq B
$$

where $\kappa_{a}$ is the construction cost per unit length of link $a$ and $u_{a}$ is the capacity enhancement of link $a . l_{a}$ is the length of link $a . B$ is the available capacity improvement budget. The left hand side of condition (16) is the total construction cost, which cannot be greater than the budget. This condition implies that when the budget is limited, not all candidate links can be improved.

For practical and physical reasons, roads or highways rarely have more than a few lanes. Therefore, BTPE includes link improvement constraints, expressed as

$$
\begin{gathered}
u_{a} \leq u_{a, \max }, \forall a \in A \\
u_{a} \geq 0, \text { are integers, } \forall a \in A
\end{gathered}
$$

where $u_{a \text { max }}$ is the maximum allowable capacity. Equation (18) is the non-negativity condition of capacity improvements together with the integer requirement.

\section{SOLUTION METHOD: ENHANCED CHEMICAL REACTION OPTIMIZATION}

\subsection{Key components of the enhanced CRO}

In this paper, the new meta-heuristic, chemical reaction optimization (CRO), is improved and employed to solve the proposed problem. This meta-heuristic mimics the process of high-energy molecules taking part in various types of elementary reactions to become the final products with stable low-energy states. It has two major components, namely, 
molecules and elementary reactions.

3.1.1 Molecules. Molecules (denoted by $M$ ) are the manipulated agents in the enhanced CRO and each has the following three properties:

1) Molecular structure $\omega$ : This represents a solution of the problem.

2) Potential energy (PE): This corresponds to the objective function value of a solution.

3) Kinetic energy (KE): It defines the measure of tolerance for a solution changing to a worse solution. This contributes to the ability of the algorithm of jumping out of local minima.

Each of the molecules can be considered to be inside a container undergoing a series of chemical reactions until each of them reaches a stable state.

3.1.2 Elementary Reactions. The core feature of the enhanced CRO is the conservation of energy in chemical reactions. Energy cannot be created or destroyed but PE and $\mathrm{KE}$ are allowed to convert into each other through a series of elementary reactions, including 1) on-wall ineffective collision; 2) decomposition; 3) inter-molecular ineffective collision and 4) synthesis.

1) On-wall ineffective collision: This mimics the reaction that a molecule hits the wall of the container. This collision is not so vigorous that only a small change in molecular structure can take place. This change can be mathematically expressed as

$$
\omega^{\prime}=N(\omega)
$$

where $N$ is the neighborhood search operator which modifies the current molecular structure $\omega$ within a small extent. $\omega^{\prime}$ is the molecular structure of the reaction product. The new molecule can be created only if it possesses a lower PE than the reactant's total energy:

$$
P E_{\omega}+K E_{\omega} \geq P E_{\omega^{\prime}}
$$

where $P E_{\omega}$ and $K E_{\omega}$ are the potential energy and kinetic energy held by the reactant whose molecular structure is represented by $\omega . P E_{\omega^{\prime}}$ is the potential energy associated with the new molecular structure $\omega^{\prime}$ after the reaction. If (20) does not hold, then no on-wall ineffective collision is allowed and the original molecule remains in the population without any change.

This reaction may result in a loss of molecular energy to the environment inside the container, leading to an exothermic reaction (i.e., the reaction that releases energy). Because of the conservation of energy, the energy released from the molecule cannot be destroyed. The enhanced CRO must develop a way to store the energy released from the molecules in case the reaction is endothermic. For this purpose, a central energy buffer, denoted by buffer, is created in the enhanced CRO, which can be interpreted as the energy in the environment obtained from exothermic reactions and supports endothermic reactions (i.e., the reactions that require energy as an input, such as decomposition).

The KE of the reactant depends on how much energy will not be lost to the environment, which is modeled by a control parameter named KELossRate. A random number $q$ from the interval [KELossRate, 1] is generated to determine the portion of KE not being lost to the environment. Hence, the $\mathrm{KE}$ of reaction product with its structure $\omega$ ' is calculated as:

$$
K E_{\omega^{\prime}}=\left(P E_{\omega}-P E_{\omega^{\prime}}+K E_{\omega}\right) \times q
$$

The lost energy is kept to the central energy buffer.

The pseudo code of this reaction is as follows:

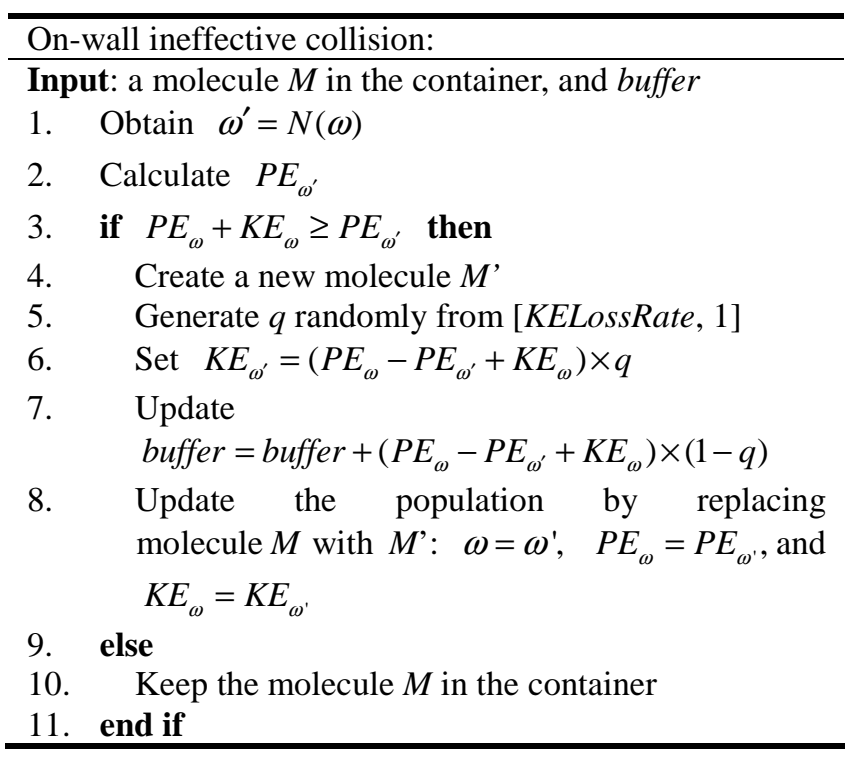

2) Decomposition: This reaction is more vigorous than the previous on-wall ineffective collision. When a molecule $M$ with structure $\omega$ hits the wall, it decomposes into two molecules with structures $\omega_{1}^{\prime}$ and $\omega_{2}^{\prime}$ respectively. Hence, the new molecular structures differ from that of the reactant molecule greatly. The structures of new molecules are defined by the decomposition operator $\mathbf{D}$ :

$$
\left[\omega_{1}^{\prime}, \omega_{2}^{\prime}\right]=\mathbf{D}(\omega)
$$

This decomposition takes place if the total PE of the two new molecules is lower than the total energy of the reactant:

$$
P E_{\omega}+K E_{\omega} \geq P E_{\omega_{1}^{\prime}}+P E_{\omega_{2}^{\prime}}
$$

As the chemical process evolves, the KE of each molecule tends to decrease in a sequence of on-wall ineffective collisions ( $\mathrm{Lam}$ and $\mathrm{Li}, 2010$ ). If the original molecule does not possess enough total energy for this transformation, the buffer can be utilized to encourage decomposition to take place, similar to an endothermic reaction intuitively. Hence, if Equation (23) does not hold, then the following criterion is checked: 


$$
P E_{\omega}+K E_{\omega}+\text { buffer } \geq P E_{\omega_{1}^{\prime}}+P E_{\omega_{2}^{\prime}}
$$

The calculation of the new KE still obeys the rule of energy conservation. The formula for determining new KE can be found in the pseudo code below:

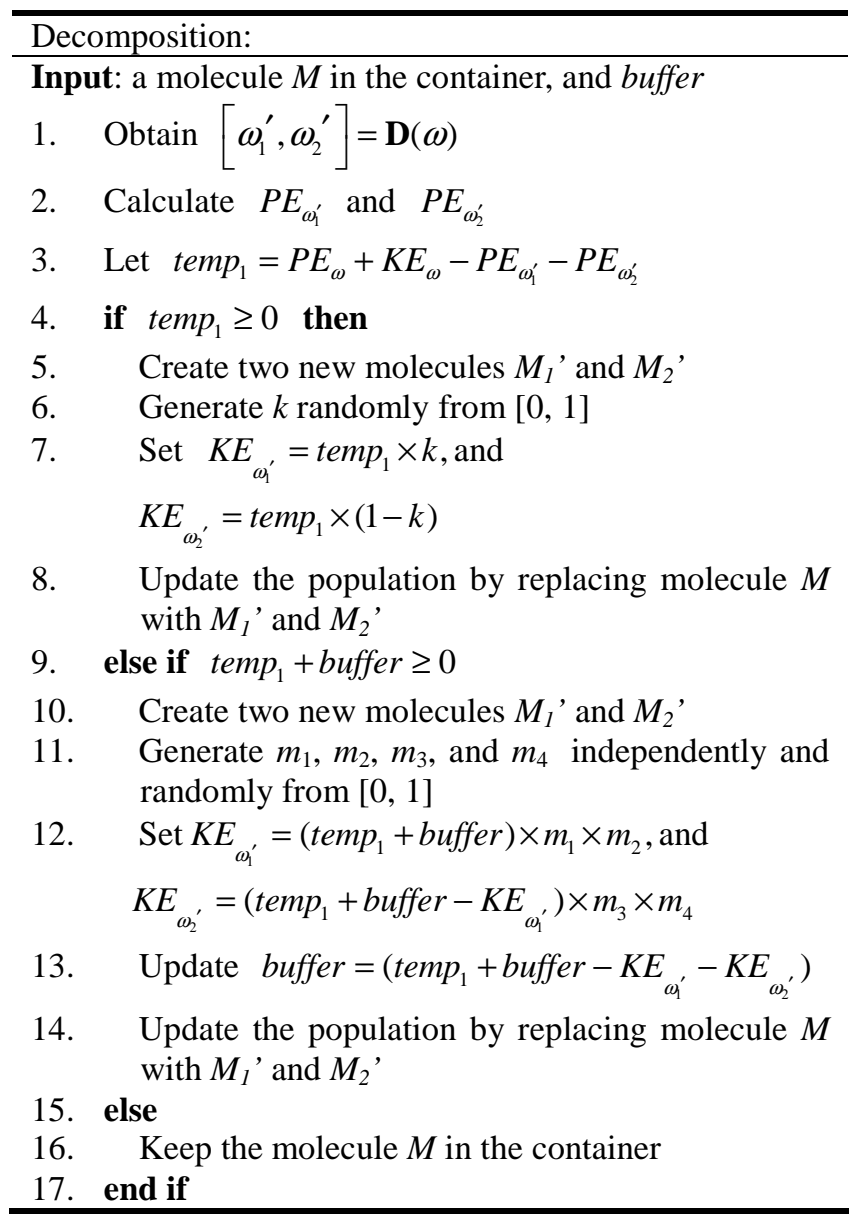

3) Inter-molecular ineffective collision: This reaction mimics the collision between two molecules and then they separate. There is no energy transformation with the central energy buffer. When two molecules collide with each other, both of them change their structures a little bit. If the new molecules possess a lower total PE than the total energy of original ones, or if

$$
P E_{\omega_{1}}+P E_{\omega_{2}}+K E_{\omega_{1}}+K E_{\omega_{2}} \geq P E_{\omega_{1}^{\prime}}+P E_{\omega_{2}^{\prime}}
$$

holds, then these two new molecules will be kept in the container. Otherwise, the original ones will be maintained.

The molecular structure change here is processed through the same neighborhood search operator $N$ as in the on-wall ineffective collision:

$$
\omega_{1}^{\prime}=N\left(\omega_{1}\right) \text { and } \omega_{2}^{\prime}=N\left(\omega_{2}\right)
$$

The calculation of KE can be found in the pseudo code of inter-molecular ineffective collision below:

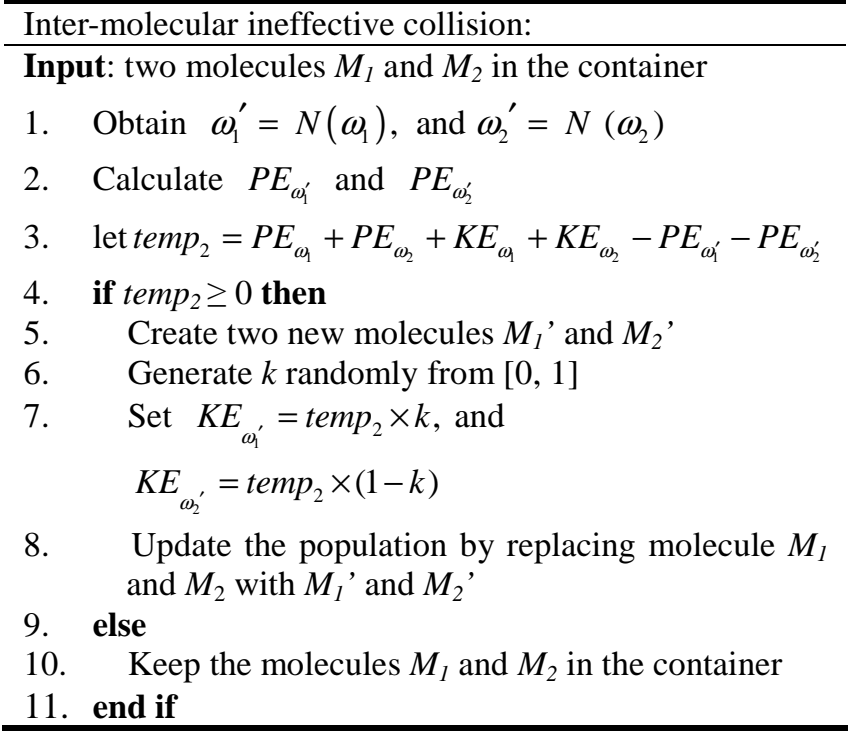

4) Synthesis: This reaction simulates the collision between two molecules and then they combine. This reaction is vigorous and the resultant molecule is very different from the original two molecules. The criterion for synthesis to occur is expressed by:

$$
P E_{\omega_{1}}+P E_{\omega_{2}}+K E_{\omega_{1}}+K E_{\omega_{2}} \geq P E_{\omega^{\prime}}
$$

The new molecular structure $\omega^{\prime}$ is obtained through the synthesis operator $S$, which is mathematically expressed as:

$$
\omega^{\prime}=S\left(\omega_{1}, \omega_{2}\right)
$$

The pseudo code of synthesis is as follows:

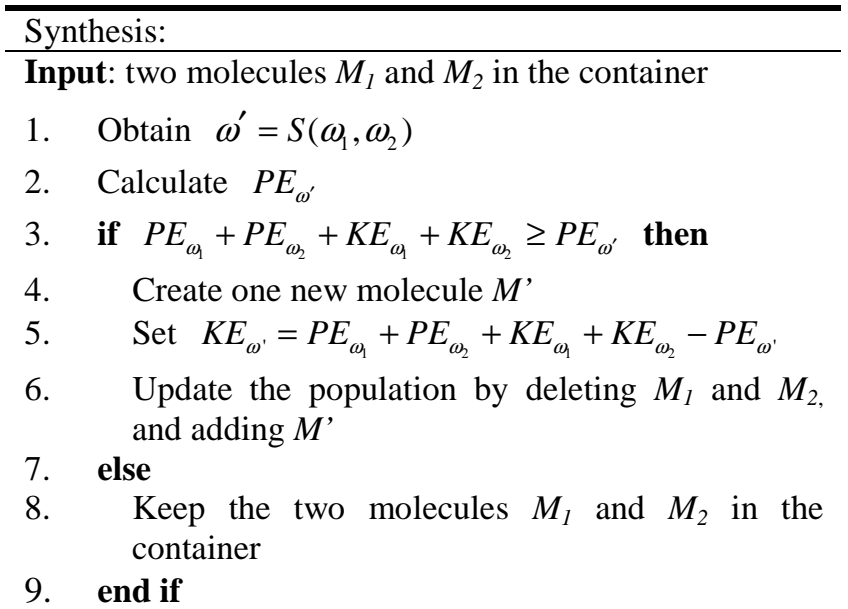

\subsection{The parameters and overall procedure of the enhanced CRO}

The parameters in the enhanced $\mathrm{CRO}$ include the following:

- InitialKE: It is the initial KE of a solution, which can be considered as the initial KE of a molecule in the container.

- $\beta$ : It is used to control the occurrence of the synthesis 
and inter-molecular ineffective collision reactions.

- popsize: It refers to the number of initial solutions generated by the enhanced CRO, which is an analogy of the number of molecules initially in the container.

- Initialbuffer: It is the initial value of central energy buffer whose default value is set to be zero (Lam et al., 2012b).

- MoleColl: It is the average fraction of inter-molecular reactions performed in the enhanced $\mathrm{CRO}$.

- KELossRate: It is the fraction of KE NOT being lost to the buffer during the reaction of an on-wall ineffective collision. It corresponds to the fraction KE not being lost to the environment due to the ineffective collision.

The overall procedure of the enhanced CRO can be divided into three main steps, namely initialization, iteration and output, in which the first step is initialization and the last step is the output stage.

In the first step, all the values of the parameters of the enhanced CRO are assigned. Then, a fixed number of initial solutions, defined by popsize, is generated. If the solution is infeasible in terms of the budget constraint, then a repairing process is carried out. Afterwards, the PE of each feasible solution is calculated and the KE of each solution is set to be InitialKE.

The second step involves a number of iterations. In each iteration, an elementary reaction is selected accordingly. Firstly, a random number $t$ from $[0,1]$ is generated. If $t$ is larger than MoleColl, the reaction will only involve one molecule at the beginning, such as on-wall ineffective collision or decomposition. Otherwise, inter-molecular ineffective collision or synthesis will take place. Then, corresponding number of molecules is selected for the reaction. Secondly, the criterion of decomposition or synthesis is checked. If one molecule is chosen and the decomposition criterion (either Condition (23) or (24)) is satisfied, then the molecule will experience a decomposition reaction. Otherwise, the on-wall ineffective collision will happen. If two molecules are chosen, then they are tested against the synthesis criterion: $\left(K E_{\omega} \leq \beta\right)$. If this criterion is satisfied, then the two selected molecules will combine through synthesis; otherwise, they will experience an inter-molecular ineffective collision. Lastly, the best solution in the current iteration is identified and compared with the best solution obtained in the last iteration. The better of the two solutions is kept. The second step is repeated until a predefined stopping criterion is met.

In the final step, the best solution found and the corresponding $\mathrm{PE}$ are reported. The flow chart of the algorithm can be found in the first paper published by Lam and $\mathrm{Li}$ (2010). The pseudo code of the enhanced CRO is as follows:
The enhanced CRO:

Input: problem specific information (objective function, constraint)

1. Assign the parameter values (InitialKE, $\beta$, KELossRate, Popsize, MoleColl)

2. Create a population

3. Repair infeasible solution

4. Determine the PE of each solution

5. Set the KE of each solution to be initialKE

6. Set the central energy buffer be buffer and assign buffer $=0$

7. do while (the stopping criteria is not satisfied)

8. Generate $t$ randomly from $[0,1]$

9. if $t>$ MoleColl then

10. Select one molecule from the population randomly

11. if decomposition criterion (Eq. (23) or (24)) is satisfied then

12. Perform the decomposition reaction

13. else

14. Perform on-wall ineffective collision

15. end if

16. else

17. Select two molecules from population randomly

18. if the synthesis criterion $\left(K E_{\omega} \leq \beta\right)$ is satisfied then

19. Perform the synthesis reaction

20. else

21. Perform inter-molecular ineffective collision

22. end if

23. end if

24. Repair infeasible solution

25. Determine the best solution up to the current iteration 26. end do

Output: the best solution and its objective function value

\subsection{Differences between the original CRO and our CRO}

There are three main differences between original CRO and ours. The first main difference between their CRO and ours is on the criterion used to determine whether decomposition or on-wall ineffective collision reaction should be carried out. Ours use conditions (23) and (24) to determine the choice. There are two advantages. First, it can ensure that no matter which reaction takes place, a new solution can be identified in each iteration. This cannot be ensured in their CRO because their CRO allows the decomposition criterion to be met but both conditions (23) and (24) not to be met. Consequently, their CRO can result in no new solution generated in some iterations, which is not beneficial especially for the case that the computation time for functional evaluation is long like ours due to solving many lower level problems. Second, we used one parameter less, which is beneficial in terms of parameter tuning.

The second main difference is that ours can be used to 
handle bilevel problems whereas the original version can only be used to handle single level optimization problems. The upper level problem is handled by the enhanced CRO framework but the lower level problem is handled by the Frank-Wolfe algorithm. The solution of the lower level problem is used to determine the objective function value of the upper level problem, and hence PE.

The third difference is that our CRO adopts a repairing strategy to handle infeasible solutions while theirs ensure the solution to be always feasible. Both PE calculation and the repairing strategy will be mentioned in the next section.

\subsection{Algorithm Implementation}

3.3.1 Solution representation. To solve our proposed network design problem with environmental consideration, the road capacity improvement strategy needs to be converted into a form which the algorithm can operate with. The decision variables in our proposed problem are the capacity improvements of candidate links. Hence, a binary number or string is used to represent whether each candidate link is improved or not. When there are $L$ decision variables, the string has $L$ bits. A bit with a value of 1 means that the link is expanded and a bit with a value of 0 means the link is not expanded (see Figure 1 for an example).

In our study, we consider two cases of improvement, namely doubling the capacity and constant capacity increment (lane addition). For the first case, expanding a link means that the capacity is doubled. This case has been considered Sharma et al. (2009). For the second case, expanding a link means that a constant of $1800 \mathrm{vph}$ is added to that link. This corresponds to adding one extra lane to the link. We can actually consider a more generalized case if we use a non-negative integer for each bit in the representation. However, this is not our main focus and hence we leave this for future study.

\begin{tabular}{|c|c|c|c|c|c|c|}
\hline & Link 1 & Link 2 & Link 3 & Link 4 & $\ldots \ldots$ & Link $L$ \\
\hline Solution 1 & 1 & 0 & 0 & 1 & $\ldots \ldots$ & 1 \\
\hline Solution 2 & 0 & 0 & 1 & 0 & $\ldots \ldots$ & 0 \\
\hline $\begin{array}{l}\vdots \\
\vdots\end{array}$ & & & & & & \\
\hline Solution M & 1 & 1 & 0 & 1 & $\ldots \ldots$ & 0 \\
\hline
\end{tabular}

Figure 1 Solution representation of $L$ candidate links

3.3.2 Initial population generation. The initialization of population utilizes a random generator. For each bit on the string, the enhanced CRO generates a random number $k$ from $[0,1]$. If $k$ is larger than 0.5 , the value of that bit is set to 1 . Otherwise, it is set to be equal to 0 .

3.3.3 Repair procedure. After the initialization, a repair process is followed. It ensures that the capacity improvement strategy represented by the solution can satisfy the budget constraint (16). This process is necessary not only in the initialization stage, but also after generating a new but infeasible solution from any reaction. The repair mechanism is to flip some of the bits of those improved links from 1 to 0 when the total improvement cost exceeds the budget. If more than one link needs to be repaired, the bit for the most expensive one will be flipped to 0 first.

3.3.4 PE evaluation and stopping criterion. After repairing all infeasible solutions, each feasible solution will be decoded to obtain the capacities of the links in the road network. Then, the UE assignment problem is solved by the Frank-Wolfe algorithm. Based on the optimal solution of the UE assignment problem and Equations (5)-(15), the objective function value is calculated.

The stopping criterion is set based on the maximum number of times required to solve the UE problem, which is 20,000. This measure is chosen rather than the maximum number of iterations normally used in other metaheuristics, including GA, because in the numerical study presented later, we want to have a fair comparison between the solution quality obtained by GA and our CRO under the same computation effort, in which the computation effort is mainly controlled by the number of times to solve the lower level problem. In our CRO, the number of solutions maintained in the population is changing over iterations, which means that the number of times using the Frank-Wolfe algorithm is changing. If we defined the stopping criterion based the maximum number of iterations, then the number of times required by our $\mathrm{CRO}$ and GA would be different.

3.3.5 Mechanism of reaction operators in the enhanced CRO. As introduced earlier in the elementary reactions in Section 3.1.2, three operators are utilized to produce new solutions. They are the Neighborhood search operator $N$, the Decomposition operator $\mathbf{D}$ and the Synthesis operator $S$. Based on the solution representation in this problem, the mechanism of these three operators are defined as follows.

The Neighborhood search operator $N$ randomly flips one bit on the string to change its value from 0 to 1 and vice versa. In Figure 2, the second bit is selected and the value is changed from 0 to 1 .

\begin{tabular}{|c|c|c|c|c|c|}
\hline \multicolumn{6}{|c|}{ Molecular structure of $M(\omega)$} \\
\hline 1 & 0 & 0 & 1 & ...... & 1 \\
\hline \multicolumn{6}{|c|}{ Molecular structure of $M^{\prime}\left(\omega^{\prime}\right)$} \\
\hline 1 & 1 & 0 & 1 & $\ldots \ldots$ & 1 \\
\hline
\end{tabular}

Figure 2 Neighborhood search operator $N$

The mechanism of the Decomposition operator $\mathbf{D}$ is defined as follows. The bit string of the original solution is separated into two segments at a random position. First (Second) segment is used to form the first (Second) segment of a new solution. The rest of the bits of each new solution is generated using the same random generator used in the 
initialization. In Figure 3, a solution with 6 bits is chosen and the second position is selected. The solution is split into two segments. The first segment has two bits and is used in the first segment of one new solution. The rest of bits in the first solution are randomly generated. The second segment has four bits and is used in the second segment of another new solution. Again, the rest of bits are randomly generated.

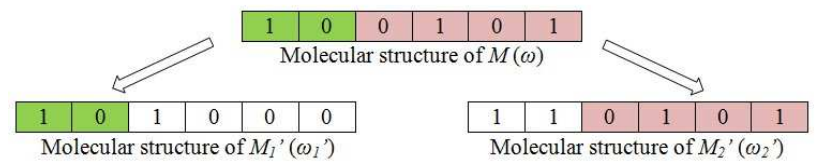

Figure 3 Decomposition operator D

The Synthesis operator $S$ is the reverse process of the Decomposition operator D. The two molecules, $M_{1}$ and $M_{2}$, are cut at the same random position and the first segment of $M_{1}$ combines with the second segment of $M_{2}$ so that the length of new solution is the same as that of the two old solutions. Figure 4 gives one example in which the second position is selected for cutting the two molecules.

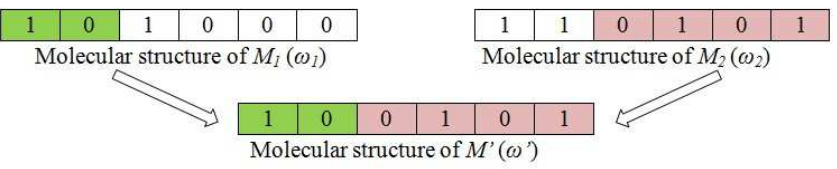

Figure 4 Synthesis operator $S$

\section{NUMERICAL STUDIES}

In this section, the testing scenarios for our CRO will firstly be provided. Then, the results of parameter tuning will be presented. Two main studies will be carried out: one is the performance comparison between our CRO and GA, and the other one is to illustrate the tradeoffs of different objectives.

\subsection{Testing scenarios}

Two road networks in the United States are selected for numerical studies. One is relatively small, Sioux Falls network and the other one is of moderate size, the Anaheim network. A brief network profile is summarized in Table 1 and the detailed configuration together with the demand matrix can be found on Bar-Gera (2012). The figures of two networks are provided in the Appendix. For each network, different demand conditions are considered, including the low demand condition, the average demand condition, and the high demand condition. Their demand matrices are obtained by multiplying the original O-D flow matrix by 0.5 , 1.0 and 2.0, respectively. $u_{a, \max }$ is set to be equal to the capacity of link $a$ and $\kappa_{a}$ is set to be free flow travel time on link $a$ divided by the length of link $a$. The VOT for TSTC is adopted as 3.88 US dollars per hour (Calfee \& Winston, 1998) for commuting. The parameter values for the noise model by Delucchi and Hsu (1996) are listed in Table 2. The monetary valuation of the specific emission factor $\phi^{k}$ provided by Matthews (1999) is listed in Table 3. All the costs are expressed as 1991 US dollars and the final costs are converted into million Hong Kong dollars in year 1991 (1 US dollar $=7.77$ in $1991 \mathrm{HK}$ dollars). A BPR function is used to model the relationship between link flow and link travel time. For each testing scenario, 20 random seeds will be generated and the average objective function value is computed.

Table 1

List of networks to be tested

\begin{tabular}{l|l|l|l|l|l}
\hline & Networks & Zones & Nodes & Links & Budget \\
\hline 1 & Sioux Falls & 24 & 24 & 76 & $\begin{array}{l}70,000 \text { (for } \\
\text { lane addition) } \\
40,000 \text { (for } \\
\text { doubling the } \\
\text { capacity) }\end{array}$ \\
\hline 2 & Anaheim & 38 & 416 & 914 & $\begin{array}{l}25,000 \text { (for } \\
\text { lane addition) }\end{array}$ \\
\hline
\end{tabular}

Table 2

Parameter input values for noise model

\begin{tabular}{|c|c|c|c|c|c|c|c|c|c|c|c|}
\hline Symbol & $H D$ & $P$ & $H V$ & $T^{\prime}$ & $T$ & $\varphi^{\prime}$ & $C_{a}$ & $\alpha$ & $B_{h}$ & $\mathrm{~d}_{\mathrm{e}}$ & $t^{*}$ \\
\hline Unit & units $/ \mathrm{mi}^{2}$ & \$/unit & $\% / \mathrm{dBA}$ & $\min$ & $\min$ & deg & & & $\mathrm{dBA}$ & Feet & $\mathrm{dBA}$ \\
\hline Value(s) & $\begin{array}{l}1290.8 \text { (Sioux Falls) } \\
4039 \text { (Anaheim) }\end{array}$ & $\begin{array}{l}3629.9 \text { (Sioux Falls) } \\
8101.3 \text { (Anaheim) }\end{array}$ & 0.0085 & 250.6 & 921.1 & 59 & 5.10 & 0.375 & 8.4 & 95.7 & 55 \\
\hline
\end{tabular}

Table 3

Monetary valuation of specific emission factors

\begin{tabular}{l|l|l|l}
\hline & $C O$ & $V O C$ & $N O_{x}$ \\
\hline$\phi^{k}$ in dollars (1991) per kg & 0.51 & 1.36 & 1.03 \\
\hline
\end{tabular}

\subsection{Parameter tuning of the enhanced CRO}

It is well known that parameter tuning can be time-consuming while parameter values are crucial for metaheuristics to obtain nearly optimal solutions. According 
to the mechanism and criteria of the occurrence of reactions, a conclusion can be drawn that InitialKE and $\beta$ have a direct and close relationship with PE, the objective function value in our study, which usually varies widely between different problems. Therefore, the fixed parameter values may not exert their best effects on optimization problems with very different objective function values.

In order to ease this situation, a normalized parameter tuning method is proposed for tuning InitialKE and $\beta$. An objective function value is computed in a trial test, assuming that there are no improvements on links. This value corresponds to the maximum objective value among all the solutions. Then, the tuning results for InitialKE and $\beta$ are expressed as the ratio of each of their values to the maximum objective value. This tuning method can simplify the tuning process for InitialKE and $\beta$ and improve the quality of solutions.

The test instance for parameter tuning was selected as the constant improvement strategy for the Sioux Falls network under the low demand condition with budget equal to 70,000, because of its calculation simplicity compared with large and congested networks. 20 random seeds were used for testing. To understand the general performance of the metaheuristic, the average value of the best objective value obtained in each run was recorded for determining parameter values.

Before the tuning process, the objective function value without any link improvement in this problem was firstly calculated, which is equal to 434792.84. Then, the initial combination for the values of the six parameters [InitialKE, $\beta$, popsize, initialbuffer, MoleColl, and KELossRate] was set to be $[1.0,0.5,200,0,0.5,0.5]$, in which the values for InitialKE and $\beta$ are normalized. During the tuning, with the values of other parameters fixed, InitialKE was first varied and the best value found was 4.0. Then, the parameter combination was changed to $[4.0,0.5,200,0,0.5,0.5]$. Then, the value of the second parameter, $\beta$, in this new combination was tuned while other values were fixed. This process was repeated until all parameter values were tuned.

The tuning results of all InitialKE, $\beta$, popsize, initialbuffer, MoleColl, and KELossRate are plotted in Figure 5, in which the $y$-axis is the average value of the best objective value obtained by each run, which is referred to as the average best objective value. The graph is not smooth in general because the enhanced CRO performs a stochastic search. However, we can still observe some reasonable trends. For example, too small or too large values for $\beta$, KELossRate and MoleColl are not good. The selected parameter values are $[4.0,2.0,100,0,0.6,0.6]$, which will be used in all the numerical studies presented later.

\subsection{Study 1: Objective function comparison}

In this study, the Sioux Falls network is used to demonstrate the importance of including environmental costs in the NDP, as well as the tradeoff between TSTC, TEC, and TNC.

To demonstrate the importance of including environmental costs in the NDP, a base case which only includes TSTC in the objective function is created for comparison with two other cases: one considers the sum of TSTT and TEC in the objective function, and another one considers the total cost (i.e., $T S T C+T E C+T N C$ ). The results are shown in Table 4 . $0.5 \mathrm{D}, 1.0 \mathrm{D}$ and $2.0 \mathrm{D}$ denote the cases of low, average, and high demand conditions, respectively.
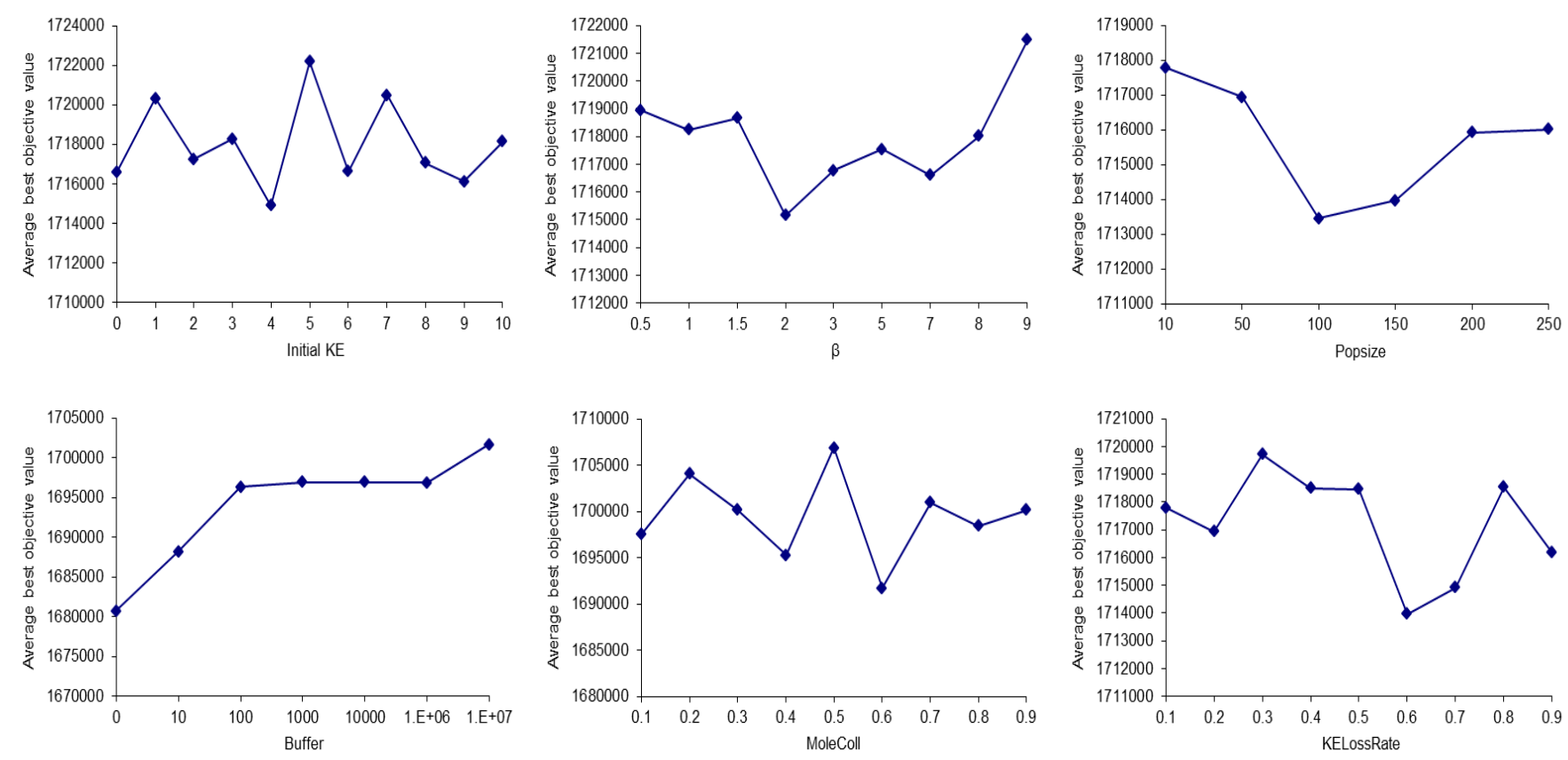

Figure 5 Parameter tuning for the enhanced CRO 
Table 4

Average cost comparisons between TSTC only and total cost $(T S T C+T E C+T N C)$

\begin{tabular}{llllll}
\hline \multirow{2}{*}{ Instance } & \multicolumn{4}{c}{ Costs (million HKD) } \\
\cline { 2 - 6 } & Base Case $($ TSTC only) & TSTC + TEC & Difference & TSTC + TEC + TNC & Difference \\
\hline $0.5 \mathrm{D}$ & 415782.42 & 418596.44 & $0.67 \%$ & 1710010.19 & $311.27 \%$ \\
$1.0 \mathrm{D}$ & 1438717.85 & 1445928.42 & $0.50 \%$ & 5189190.17 & $260.68 \%$ \\
$2.0 \mathrm{D}$ & 19971509.22 & 20031246.20 & $0.30 \%$ & 24536201.70 & $22.86 \%$ \\
\hline
\end{tabular}

As shown in Table 4, the sum of the costs increases with the number of the cost components included. The increase is very obvious especially when the noise cost is included. The maximum percentage increase is more than 3.1 times, which is quite large and should not be ignored. This result demonstrates the need to incorporate environmental considerations into the NDP.

Besides the significant effects on the magnitude of costs, the incorporation of environmental concerns also has a great influence on the network design solution. Table 5 provides the final design solutions under the low demand situation and different objective functions.

\section{Table 5}

Final design under different objective functions

\begin{tabular}{c|c|c|c}
\hline & TSTC & TSTC+TEC & $\begin{array}{c}\text { TSTC+TEC } \\
+T N C\end{array}$ \\
\cline { 2 - 4 } & 19 & 19 & \\
& 29 & 29 & 2 \\
& 34 & 34 & 9 \\
& 35 & 35 & 11 \\
Links to be & 40 & 40 & 18 \\
improved & 42 & 42 & 26 \\
(Link No.) & 53 & 48 & 33 \\
& 55 & 53 & 36 \\
& 58 & 55 & 45 \\
& 65 & 57 & 46 \\
& 66 & 65 & 49 \\
& 69 & 69 & 53 \\
& 70 & 70 & 58 \\
& 72 & 72 & 60 \\
& 74 & 74 & 74 \\
& 75 & 75 & \\
\hline Total costs & 414254.85 & 417060.64 & 1698123.28 \\
\hline
\end{tabular}

It can be seen that the final design for the scenario considering noise cost is significantly different from those without noise cost (i.e., TSTC or TSTC + TEC). This is because the noise cost is not monotonic with respect to average speed and link flow, compared to the monotonic relationship between travel time cost and link flow. Although the emission cost possesses certain nonlinearity with respect to flow as well, the magnitude of emission cost is relatively minor compared to TSTC and hence fails to change the design under the consideration of TSTC. Therefore, the designs without considering noise cost are the same.

In the following analysis, the tradeoff between the three cost components is explored. We constructed a model with the objective function considering only one cost component, and determined the best objective value. At the same time, we obtained the values of other cost components. The results are shown in Table 6 below. The arrows pointing up or down indicate that the value increases or decreases compared with the base case (TSTC only). The asterisk (*) in front of the number denotes that this is the smallest value in the row.

Table 6

Cost comparison between different single objective functions

\begin{tabular}{lclll}
\hline \multirow{2}{*}{ Instance } & $\begin{array}{c}\text { Component } \\
\text { values }\end{array}$ & TSTC & TEC & TNC \\
\cline { 3 - 5 } & & & & \\
\hline \multirow{2}{*}{$0.5 \mathrm{D}$} & TSTC & $* 414254.85$ & $420761.69 \uparrow$ & $426452.67 \uparrow$ \\
& TEC & 2805.8 & $* 2766.69 \downarrow$ & $2776.86 \downarrow$ \\
& TNC & 1465563.5 & $1291911.47 \downarrow$ & $* 1266122.51 \downarrow$ \\
\hline \multirow{2}{*}{$1.0 \mathrm{D}$} & TSTC & $* 1428817.98$ & $1438903.57 \uparrow$ & $1602971.4 \uparrow$ \\
& TEC & 7165.62 & $* 7146.68 \downarrow$ & $7500.76 \uparrow$ \\
\hline \multirow{2}{*}{$2.0 \mathrm{D}$} & TNC & 3925517.87 & $3833606.63 \downarrow$ & $* 3608278.20 \downarrow$ \\
\hline & TSTC & $* 19509685.92$ & 19509685.92 & $20214524.65 \uparrow$ \\
& TEC & 58469.82 & $* 58469.82$ & $60361.3 \uparrow$ \\
\hline & TNC & 4399355.34 & 4399355.34 & $* 4375468.63 \downarrow$ \\
\hline
\end{tabular}

Some important and interesting findings emerge from the results. Firstly, the three costs cannot be simultaneously optimized in general. As indicated by the arrows in one scenario, when the objective component value is minimized, the costs of the other two components do not necessarily decrease and they usually increase. This implies that there are tradeoffs between designing a network for minimal total travel time, minimal total emission cost, and minimal total noise cost. This is because the relationship between emission factors and $L_{e q}$ are not monotonic with respect to speed and link flow but the relationship between travel time and link flow is.

Secondly, all the cost components as well as the total cost increase as demand on the network increases. The increase 
amplitude of TSTC is much larger than those of TEC and $T N C$. This implies that in our modeling framework, TSTC is more sensitive to the link flow change than others.

\subsection{Study 2: The enhanced CRO and GA performance comparison}

To test the performance of our CRO solving BTPE, GA is used as a comparison. GA is a relatively classic metaheuristic in solving complicated, often NP hard, optimization problems. Besides previously listed BTPEs solved by GA, it also has a wide range of successful applications in various fields including transportation engineering (e.g., Putha et al., 2012), structural engineering (e.g., Adeli and Cheng, 1993; Adeli and Kumar, 1999; Adeli and Sarma, 2006; Kim and Adeli, 2001; Hsiao et al., 2012; Marano et al., 2011; Sarma and Adeli, 2001, 2002; Sgambi et al., 2012) and electrical and electronic engineering (e.g., Adeli and Hung, 1995; Hung and Adeli, 1994). Similar to our CRO, GA has a framework in which other techniques can be incorporated to produce a hybrid that reaps the best performance on different problems (e.g., Adeli and Cheng, 1994a,b; Adeli and Kumar, 1995a,b; Sarma and Adeli, 2000a,b; Jiang and Adeli, 2008).

To carry out a fair comparison, the GA parameters were also fine-tuned. The selected value of mutation probability and popsize were tuned as 0.01 and 100, respectively. The solution representation, the initial solution generation procedure and the repairing procedure are identical to those of the enhanced CRO. The fitness of each solution is equal to PE. All parents were allowed to reproduce offspring. Roulette wheel selection was adopted for the reproduction process. The crossover operator adopted is one point crossover with the crossover rate of 1 at each bit. In this study, two cases were tested for the performance comparison of the enhanced CRO and GA: doubling the capacity and the constant capacity increment.

4.4.1 Doubling the capacity. In this scenario, the Sioux Falls network with different demand conditions was used with the budget equal to 40,000 (SD case). The results of 20 seeds are plotted in the Figure 6 below and the summary of detailed numerical results is given in Table 7 . The value in bold is the best value of the two algorithms for the criteria considered. It is obviously observed that, our CRO has a better overall performance on this scenario with lower average objective function values and smaller standard deviations mostly.

The convergence plots of our CRO and GA using a random seed on the Sioux Falls network are provided in Figure 9. From the convergence comparison plot, our CRO shows a relatively faster speed to converge and reaches a better objective function value within 3000 lower level problem evaluations.

4.4.2 Lane addition. In this scenario, the Sioux Falls network (SC case) and the Anaheim network (AC case) with different demand conditions were both tested. The budget settings for these two network applications are provided in Table 1. The best objective function values are plotted in Figure 7 and Figure 8 respectively. The detailed numerical results can also be referred to Table 7 as well. Our CRO shows better performance to search for good solutions for the smaller network.
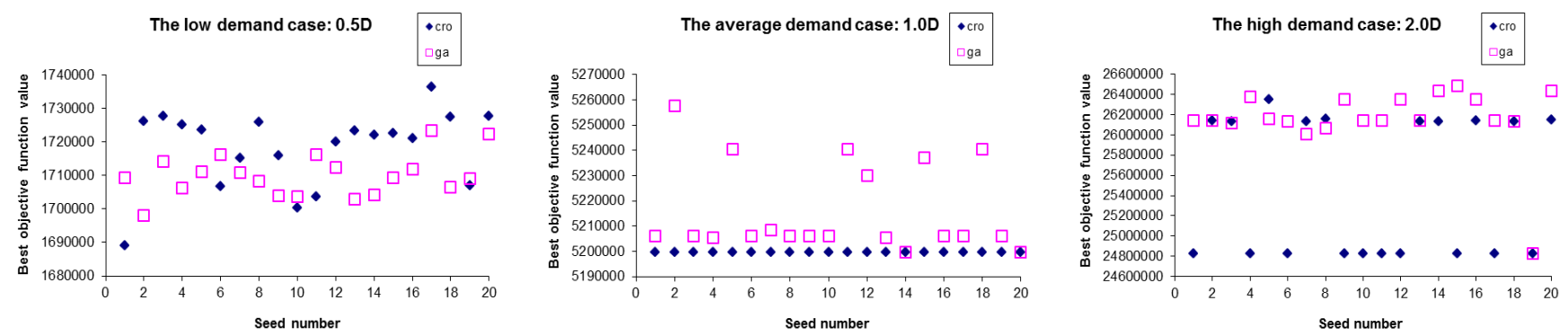

Figure 6 Best objective function values for the Sioux Falls network scenario (doubling the capacity)
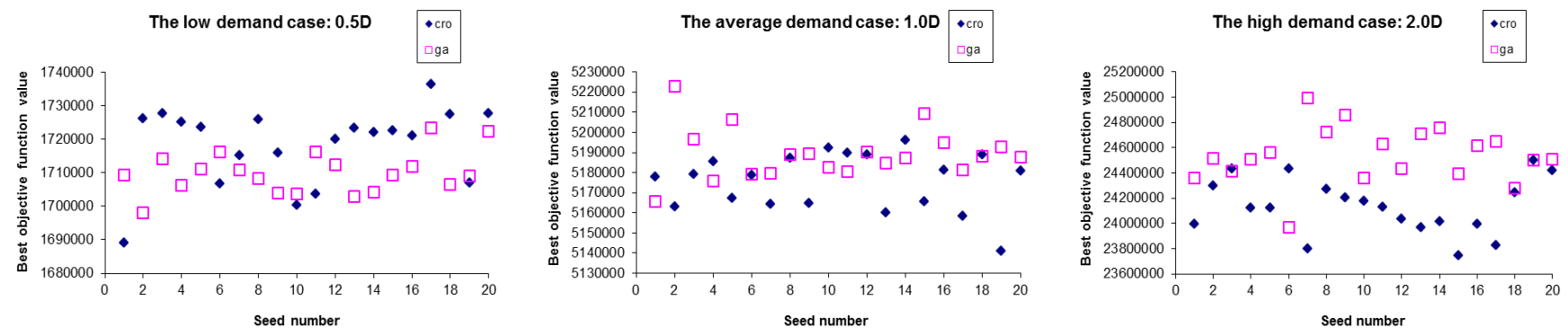

Figure 7 Best objective function values for the Sioux Falls lane addition case 

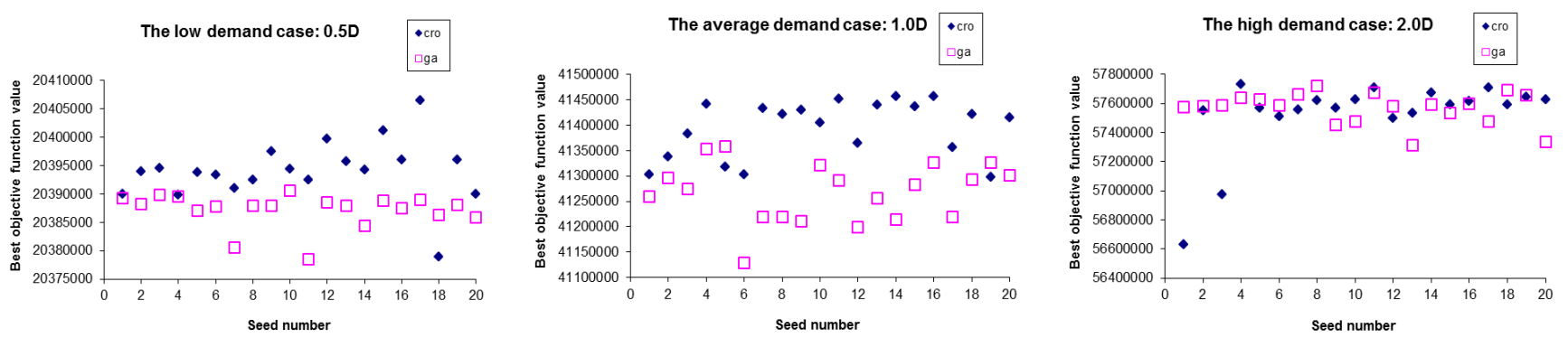

Figure 8 Best objective function values for the Anaheim lane addition scenario
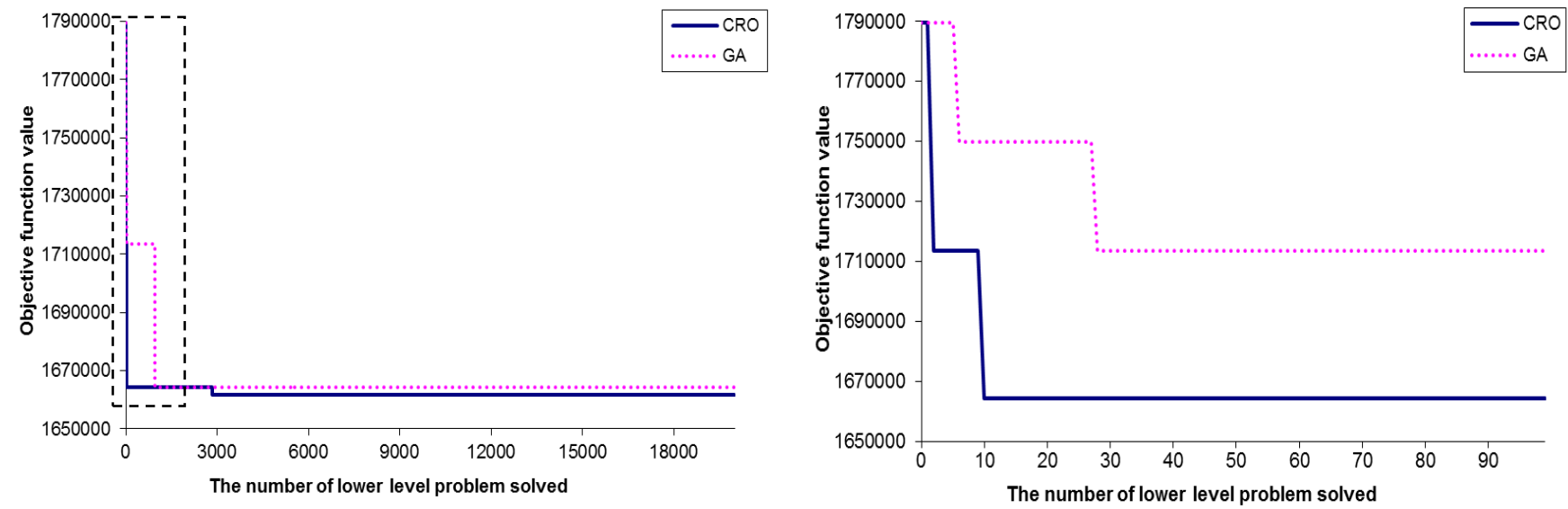

Figure 9 Convergence plots of the enhanced CRO and GA and an enlarged view within the dashed box

(Doubling the capacity, the low demand case: 0.5D.)

Table 7

Statistical results and computation time

\begin{tabular}{|c|c|c|c|c|c|c|c|c|c|c|}
\hline \multirow[b]{2}{*}{ Network } & \multirow[b]{2}{*}{$\begin{array}{l}\text { Demand } \\
\text { conditions }\end{array}$} & \multirow{2}{*}{$\begin{array}{l}\text { No. of } \\
\text { traffic } \\
\text { assignment } \\
\text { problems } \\
\text { solved }\end{array}$} & \multicolumn{4}{|c|}{ Enhanced CRO } & \multicolumn{4}{|c|}{$G A$} \\
\hline & & & Міпітит & Mean & $\begin{array}{l}\text { Standard } \\
\text { Deviation }\end{array}$ & Time $(s)$ & Міпітит & Mean & $\begin{array}{l}\text { Standard } \\
\text { Deviation }\end{array}$ & Time $(s)$ \\
\hline \multirow{3}{*}{$\mathrm{SC}$} & $0.5 \mathrm{D}$ & 20,000 & 1688986.15 & 1718373.35 & 11527.81 & 636.08 & 1698123.28 & 1710010.19 & 6367.99 & 679.38 \\
\hline & $1.0 \mathrm{D}$ & 20,000 & 5140944.20 & 5175589.20 & 14318.84 & 1601.06 & 5165790.23 & 5189189.94 & 12693.01 & 1661.80 \\
\hline & $2.0 \mathrm{D}$ & 20,000 & 23748654.72 & 24136630.09 & 217714.57 & 2275.99 & 23967511.09 & 24536201.70 & 224751.76 & 2384.77 \\
\hline \multirow{3}{*}{ SD } & $0.5 \mathrm{D}$ & 20,000 & 1657075.53 & 1662193.54 & 1621.33 & 696.00 & 1661778.99 & 1665569.29 & 7713.83 & 744.80 \\
\hline & $1.0 \mathrm{D}$ & 20,000 & 5199695.85 & 5199695.85 & 0.00 & 1631.73 & 5199695.85 & 5215980.87 & 17568.83 & 1754.03 \\
\hline & $2.0 \mathrm{D}$ & 20,000 & 24827783.76 & 25494023.55 & 685112.66 & 2246.61 & 24827783.76 & 26153756.07 & $\mathbf{3 4 1 8 3 4 . 3 3}$ & 2396.09 \\
\hline \multirow{3}{*}{$\mathrm{AC}$} & $0.5 \mathrm{D}$ & 20,000 & 20378950.82 & 20394079.59 & 5402.73 & 32473.59 & 20378418.87 & 20387157.82 & 3000.03 & 43885.69 \\
\hline & $1.0 \mathrm{D}$ & 20,000 & 41297460.72 & 41393602.60 & 55942.77 & 63225.6 & 41128122.40 & 41267375.65 & 59158.11 & 66071.48 \\
\hline & $2.0 \mathrm{D}$ & 20,000 & 57685492.75 & 57525700.48 & 261244.03 & 90548.05 & 57313315.30 & 57567537.14 & 109019.43 & 95833.48 \\
\hline
\end{tabular}


To have a more accurate comparison, a t-test with $95 \%$ confidence interval on the difference in means of total costs $(T S T C+T E C+T N C)$ obtained by the enhanced CRO and GA was carried out. An illustration is provided in Table 8. To present the results concisely, the summary of the conclusion is provided in Table 9, using "s+", "s-" and " $\approx$ " to indicate that the enhanced CRO performs significantly better than, significantly worse than and comparably with GA respectively.

Table 8

Results of t-test of Sioux Falls lane addition case (1.0D)

\begin{tabular}{lll}
\hline Difference in means $($ CRO-GA) & $t$-Value & Probability \\
\hline-13600.74 & -3.718 & 0.001 \\
\hline
\end{tabular}

Table 9

Results of t-tests with $95 \%$ confidence interval on the difference in means between the enhanced CRO and GA

\begin{tabular}{|c|c|c|c|c|}
\hline Instance & Network & $\begin{array}{l}\text { Traffic } \\
\text { condition }\end{array}$ & $C R O$ & $G A$ \\
\hline \multirow{3}{*}{$\begin{array}{l}\text { Double } \\
\text { capacity } \\
\text { improvement }\end{array}$} & \multirow{3}{*}{$\begin{array}{l}\text { Sioux } \\
\text { Falls }\end{array}$} & $0.5 \mathrm{D}$ & s+ & S- \\
\hline & & $1.0 \mathrm{D}$ & s+ & S- \\
\hline & & $2.0 \mathrm{D}$ & s+ & S- \\
\hline \multirow{6}{*}{$\begin{array}{l}\text { Constant } \\
\text { capacity } \\
\text { improvement }\end{array}$} & \multirow{3}{*}{$\begin{array}{l}\text { Sioux } \\
\text { Falls }\end{array}$} & $0.5 \mathrm{D}$ & S- & s+ \\
\hline & & $1.0 \mathrm{D}$ & S+ & S- \\
\hline & & $2.0 \mathrm{D}$ & $\mathrm{s}+$ & S- \\
\hline & \multirow{3}{*}{ Anaheim } & $0.5 \mathrm{D}$ & S- & $\mathrm{s}+$ \\
\hline & & $1.0 \mathrm{D}$ & S- & $\mathrm{s}+$ \\
\hline & & $2.0 \mathrm{D}$ & $\approx$ & $\approx$ \\
\hline
\end{tabular}

According to the No-Free-Lunch (NFL) theorem (Wolpert and Macready, 1997), our CRO must perform comparably with other algorithms on average, but it can outperform other meta-heuristics when matching to the right problem. In our study, the enhanced CRO performed significantly better on solving the case of doubling the capacity. Moreover, the enhanced CRO achieved better solutions in more than half of the test cases and has shorter computation time among the entire scenarios.

\section{CONCLUSIONS}

This research proposes a new bilevel discrete network design problem that considers both vehicle emission and noise costs and the total system travel time cost simultaneously. An enhanced $\mathrm{CRO}$ with a repair procedure is developed to solve the problem. A normalized parameter tuning method is also proposed for CRO implementation in this study. Numerical studies are set up to illustrate the performance of our CRO and the properties of the problem. The key findings and implications are as follows:

1. External environmental costs caused by the traffic can be substantial and cannot be neglected. Moreover, environmental concerns can influence the network design pattern significantly. Hence, it is important for transportation network planners to incorporate the environmental costs in the NDP and minimize them.

2. Minimizing the total system travel time cost does not necessarily minimize the environmental costs at the same time. There are tradeoffs between these objectives. The transportation network planners need to consider the tradeoff carefully when improving transportation networks.

3. Within 20,000 times of solving the traffic assignment problem, our CRO is able to obtain a better objective function value than GA in some cases (e.g., in most of the Sioux Fall network cases with 24 nodes and 76 links) whereas they can have comparable performance on other cases (such as for the congested network with the numbers of zones, links and nodes of 38, 416, and 914 respectively). It is no harm to use the CRO approach to solve other problems that have been solved by GA but not CRO in the future. Perhaps, better solutions can be obtained.

We believe that the above key findings and implications are useful for future research, and can open up more new research directions For example, we can compare the performance of the proposed solution method and evolutionary strategy (e.g., Jafarkhani and Masri, 2011) on solving BTPE.

\section{ACKNOWLEDGEMENTS}

The work described in this paper was jointly supported by a grant from the National Natural Science Foundation of China (71271183), a grant (201011159026) from the University Research Committee, and a Research Postgraduate Studentship from the University of Hong Kong. The authors are grateful to the three reviewers for their constructive comments.

\section{REFERENCES}

Adeli, H. \& Cheng, N.-T. (1993), Integrated genetic algorithm for optimization of space structures, Journal of Aerospace Engineering, 6(4), 315-328.

Adeli, H. \& Cheng, N.-T. (1994a), Augmented Lagrangian genetic algorithm for structural optimization, Journal of Aerospace Engineering, 7(1), 104-118.

Adeli, H. \& Cheng, N.-T. (1994b), Concurrent genetic algorithms for optimization of large structures, Journal of Aerospace Engineering, 7(3), 276-296.

Adeli, H. \& Hung, S. L. (1995), Machine Learning - Neural Networks, Genetic Algorithms, and Fuzzy Sets, John Wiley and Sons, New York.

Adeli, H. \& Kumar, S. (1995a), Distributed genetic algorithms for structural optimization, Journal of Aerospace Engineering, 8(3), 156-163. 
Adeli, H. \& Kumar, S. (1995b), Concurrent structural optimization on a massively parallel supercomputer, Journal of Structural Engineering, 121(11), 1588-1597.

Adeli, H. \& Kumar, S. (1999), Distributed Computer-Aided Engineering for Analysis, Design, and Visualization, CRC Press, Boca Raton, Florida.

Adeli, H. \& Sarma, K. (2006), Cost Optimization of Structures - Fuzzy Logic, Genetic Algorithms, and Parallel Computing, John Wiley and Sons, West Sussex, United Kingdom.

Bar-Gera, H. (2012), Transportation Network Test Problems, http://www.bgu.ac.il/ bargera/tntp/ (Accessed on 5 May 2012).

Calfee, J. \& Winston, C. (1998), The value of auto mobile travel time: implications for congestion policy, Journal of Public Economics, 96, 83-102.

Chen, A. \& Xu, X. (2012), Goal programming approach to solving the network design problem with multiple objectives and demand uncertainty, Expert Systems with Applications, 39(4), 4160-4170.

Chen, A., Chootinan, P. \& Wong, S.C. (2006), New reserve capacity model of a signal-controlled road network, Transportation Research Record, 1964, 35-41.

Chen, A., Kim, J., Lee, S. \& Kim, Y. (2010), Stochastic multi-objective models for network design problem under demand uncertainty, Expert Systems with Applications, 37(2), 1608-1619.

Delucchi, M. \& Hsu, S. L. (1996), The external damage cost of direct noise from motor vehicles, Report \#14 in the series: The annualized social cost of motor-vehicle use in the United States, based on 1990-1991 data.

Duthie, J. \& Waller, S. T. (2008), Incorporating environmental justice measures into equilibrium-based network design. Transportation Research Record, 2089, 58-65.

Ferguson, E. M., Duthie, J. \& Waller, S. T. (2010), Network Methods for Project Selection based on Optimizing Environmental Impact, Research Report SWUTC/10/161026-1 (Austin: University of Texas at Austin).

Ferguson, E. M., Duthie, J. \& Waller, S. T. (2012), Comparing delay minimization and emissions minimization in the network design problem, Computer-Aided Civil and Infrastructure Engineering, 27(4), 288-302.

Friesz, T. L., Tobin, R. L., Cho, H. J. \& Mehta, N. J. (1990), Sensitivity analysis based heuristic algorithms for mathematical programs with variational inequality constraints, Mathematical Programming, 48, 265-284

Hawas, Y. E. (2011), An integrated simulation-based fuzzy logic model for real-time traffic signal control, Transportmetrica, 7(4), 247-278.

Hsiao, F. Y., Wang, S. S., Wang, W. C., Wen, C. P. \& Yu, W.
D. (2012), Neuro-Fuzzy cost estimation model enhanced by fast messy genetic algorithms for semiconductor hookup construction, Computer-Aided Civil and Infrastructure Engineering, 27(10), 764-781.

Huang, K., Zhang, J., He, M. \& Zhu, J. (2009), Bi-level programming model of urban traffic network considering noise pollution control, in Proceedings of the 2nd International Conference on Transportation Engineering (July 25-27, 2009 Chengdu, China), 3399-3404.

Hung S.L. \& Adeli, H. (1994), A parallel genetic/neural network learning algorithm for MIMD shared memory machines, IEEE Transactions on Neural Networks, 5(6), 900-909.

Jafarkhani, R. \& Masri, S. F. (2011), Finite element model updating using evolutionary strategy for damage detection, Computer-Aided Civil and Infrastructure Engineering, 26(3), 207-224.

Jia, P., Kato, H. \& Hayashi, Y. (2009), Road network optimization model with consideration of dynamic changes in long term evaluation for developing cities, in Proceedings of the 88th Transportation Research Board Annual Meeting.

Jiang, X. \& Adeli, H. (2008), Neuro-genetic algorithm for nonlinear active control of highrise buildings, International Journal for Numerical Methods in Engineering, 75(8), 770-786.

Karoonsoontawong, A. \& Waller, S. T. (2006), Dynamic continuous network design problem: linear bi-level programming and metaheuristic approaches, Transportation Research Record, 1964, 104-117.

Kim, B. J. \& Kim, W. (2006), An equilibrium network design model with a social cost function for multimodal networks. Annual on Regional Science, 40, 473-491.

Kim, H. \& Adeli, H. (2001), Discrete cost optimization of composite floors using a floating point genetic algorithm, Engineering Optimization, 33(4), 485-501.

Lam, A. Y. S. \& Li, V. O. K. (2010), Chemical-reaction-inspired metaheuristic for optimization, IEEE Transactions on Evolutionary Computation, 14(3), 381-399.

Lam, A. Y. S., Li, V. O. K. \& Wei, Z. (2012a), Chemical reaction optimization for the fuzzy rule learning problem, Proceedings of IEEE Congress on Evolutionary Computation (IEEE CEC 2012), Brisbane, Australia.

Lam, A. Y. S., Li, V. O. K. \& Yu, J. Q. (2012b), Real-coded chemical reaction optimization, IEEE Transactions on Evolutionary Computation, 16(3), 339 - 353.

Li, Z. C., Lam, W. H. K., Wong, S. C. \& Sumalee, A. (2012), Environmentally sustainable toll design for congested road networks with uncertain demand, International Journal of Sustainable Transportation, 6(3), 127-155.

Lo, H. K. \& Szeto, W. Y. (2009), Time-dependent Transport 
Network Design under Cost-recovery. Transportation Research Part B, 43(1), 142-158.

Long, J. C., Gao, Z. Y., Zhang, H. Z. \& Szeto, W.Y. (2010), A turning restriction design problem in urban road networks, European Journal of Operational Research, 206(3), 569-578.

Marano, G. C., Quaranta, G. \& Monti, G. (2011), Modified genetic algorithm for the dynamic identification of structural systems using incomplete measurements, Computer-Aided Civil and Infrastructure Engineering, 26(2), 92-110.

Matthews, H. S. (1999), The External Costs of Air Pollution and the Environmental Impact of the Consumer in the U.S. Economy, Ph.D. thesis, Carnegie Mellon University, Pittsburg, PA, USA.

Meng, Q. \& Yang, H. (2002), Benefit distribution and equity in road network design, Transportation Research Part $B, 36(1), 19-35$.

Meng, Q., Yang, H. \& Bell, M. G. H. (2001), An equivalent continuously differentiable model and a locally convergent algorithm for the continuous network design problem, Transportation Research Part B, 35(1), 83-105.

Miandoabchi, E., Zanjirani Farahani, R. \& Szeto, W. Y. (2012a), Bi-objective bimodal urban road network design using hybrid metaheuristics, Central European Journal of Operations Research, 20(4), 583-621.

Miandoabchi, E., Zanjirani Farahani, R., Dullaert, W. \& Szeto, W. Y. (2012b), Hybrid evolutionary metaheuristics for concurrent multi-objective design of urban road and public transit networks, Network for Spatial Economics, 12 (3), 441-480.

Ng, M. W. \& Waller, S. T. (2009a), Reliable system optimal network design: a convex mean-variance type model with implicit chance constraints, Transportation Research Record, 2090, 68-74.

Ng, M. W. \& Waller, S. T. (2009b), The evacuation optimal network design problem: model formulation and comparisons, Transportation Letters: The International Journal of Transportation Research, 1(2), 111-119.

Ng, M. W., Lin, D.-Y \& Waller, S. T. (2009), Optimal long-term infrastructure maintenance planning accounting for traffic dynamics, Computer-Aided Civil and Infrastructure Engineering, 24(7), 459-469.

Penic, M. A. \& Upchurch, J. (1992), TRANSYT-7F: Enhancement for fuel consumption, pollution emissions and user costs, Transportation Research Record, 1360, 104-111.

Plevris, V. \& Papadrakakis, M. (2011), A hybrid particle swarm - gradient algorithm for global structural optimization, Computer-Aided Civil and Infrastructure Engineering, 26(1), 48-68.

Putha, R., Quadrifoglio, L. \& Zechman, E. (2012), Comparing ant colony optimization and genetic algorithm approaches for solving traffic signal coordination under oversaturation conditions, Computer-Aided Civil and Infrastructure Engineering, 27(1), 14-28.

Qiu, Y. \& Chen, S. (2007), Bi-level programming for continuous network design of comprehensive transportation system based on external optimization, in Proceedings of 2007 IEEE International Conference on Grey Systems and Intelligent Services, GSIS 2007, (November 18 - 20, 2007 Nanjing, China), 1186-1190.

Sarma, K. \& Adeli, H. (2000a), Fuzzy genetic algorithm for optimization of steel structures, Journal of Structural Engineering, 126(5), 596-604.

Sarma, K. \& Adeli, H. (2000b), Fuzzy discrete multicriteria cost optimization of steel structures, Journal of Structural Engineering, 126(11), 1339-1347.

Sarma, K.C. \& Adeli, H. (2001), Bi-level parallel genetic algorithms for optimization of large steel structures, Computer-Aided Civil and Infrastructure Engineering, 16(5), 295-304.

Sarma, K.C. \& Adeli, H. (2002), Life-cycle cost optimization of steel structures, International Journal for Numerical Methods in Engineering, 55(12), 1451-1462.

Sgambi, L., Gkoumas, K. \& Bontempi, F. (2012), Genetic algorithms for the dependability assurance in the design of a long span suspension bridge, Computer-Aided Civil and Infrastructure Engineering, 27(9), 655-675

Sharma, S. \& Mathew, T. V. (2011), Multiobjective network design for emission and travel-time trade-off for a sustainable large urban transportation network, Environmental and Planning B: Planning and Design, 38(3), 520-538.

Sharma, S., Ukkusuri, S. V. \& Mathew, T. V. (2009), Pareto optimal multiobjective optimization for robust transportation network design problem, Transportation Research Record, 2090, 95-104.

Shepherd, S. (2013), Toll competition and dynamic toll-setting strategies, International Journal of Sustainable Transportation, 7(3), 186-203.

Szeto, W. Y. \& Jiang, Y. (2012), A hybrid artificial bee colony algorithm for transit network design, Transportation Research Record, 2284, 47-56.

Szeto, W. Y. \& Lo, H. K. (2006), Transportation network improvement and tolling strategies: the issue of intergeneration equity, Transportation Research Part A, 40(3), 227-243.

Szeto, W. Y. \& Lo, H. K. (2008), Time-dependent transport network improvement and tolling strategies, Transportation Research Part A, 42(2), 376-391.

Szeto, W. Y. \& Wu, Y. Z. (2011), A simultaneous bus route design and frequency setting problem for Tin Shui Wai, Hong Kong, European Journal of Operational Research, 209(2), 141-155.

Szeto, W. Y., Jaber, X. Q. \& O’Mahony, M. (2010), Time-dependent discrete network design frameworks 
considering land use, Computer-Aided Civil and Infrastructure Engineering, 25(6), 411-426.

Szeto, W. Y., Jaber, X. Q. \& Wong, S. C. (2012), Road network equilibrium approaches to environmental sustainability, Transport Reviews: A Transnational Transdisciplinary Journal, 32(4), 491-518.

Szeto, W. Y., Wu, Y. Z. \& Ho, S. C. (2011), An artificial bee colony algorithm for the capacitated vehicle routing problem, European Journal of Operational Research, 215(1), 126-135.

Wang, D. Z. W. \& Lo, H. K. (2010), Global optimum of the linearized network design problem with equilibrium flows, Transportation Research Part B, 44(4), 482-492.

Wismans, L. J. J., Berkum, E. C. V. \& Bliemer, M. C. J. (2011), Comparison of evolutionary multi objective algorithms for the dynamic network design problem, Proceedings of the 2011 International Conference on Networking, Sensing and Control, 275-280.

Wolpert, D. H. \& Macready, W. G. (1997), No free lunch theorems for optimization, IEEE Transactions on Evolutionary Computation, 1(1), 67-82.

Xu, J., Lam, A. Y. S. \& Li, V. O. K. (2011), Stock portfolio selection using chemical reaction optimization, Proceedings of International Conference on Operations Research and Financial Engineering (ICORFE 2011), Paris, France, 458-463.

$\mathrm{Xu}$, T., Wei, H. \& Wang, Z. (2009), Study on continuous network design problem using simulated annealing and genetic algorithm, Expert Systems with Applications, 36(2), 1322-1328.

Yang, H. \& Bell, M. G. H. (1998), Models and algorithms for road network design: a review and some new developments, Transport Reviews: A Transnational Transdisciplinary Journal, 18(3), 257-278.

Yu, J. J. Q., Li, V. O. K. \& Lam, A. Y. S. (2012), Sensor deployment for air pollution monitoring using public transportation system, Proceedings of IEEE Congress on Evolutionary Computation (IEEE CEC 2012), Brisbane, Australia.

Zhang, H. \& Gao, Z. (2007), Two-way road network design problem with variable lanes, Journal of Systems Science and Systems Engineering, 16(1), 50-61.

\section{APPENDIX}

Sioux Falls network

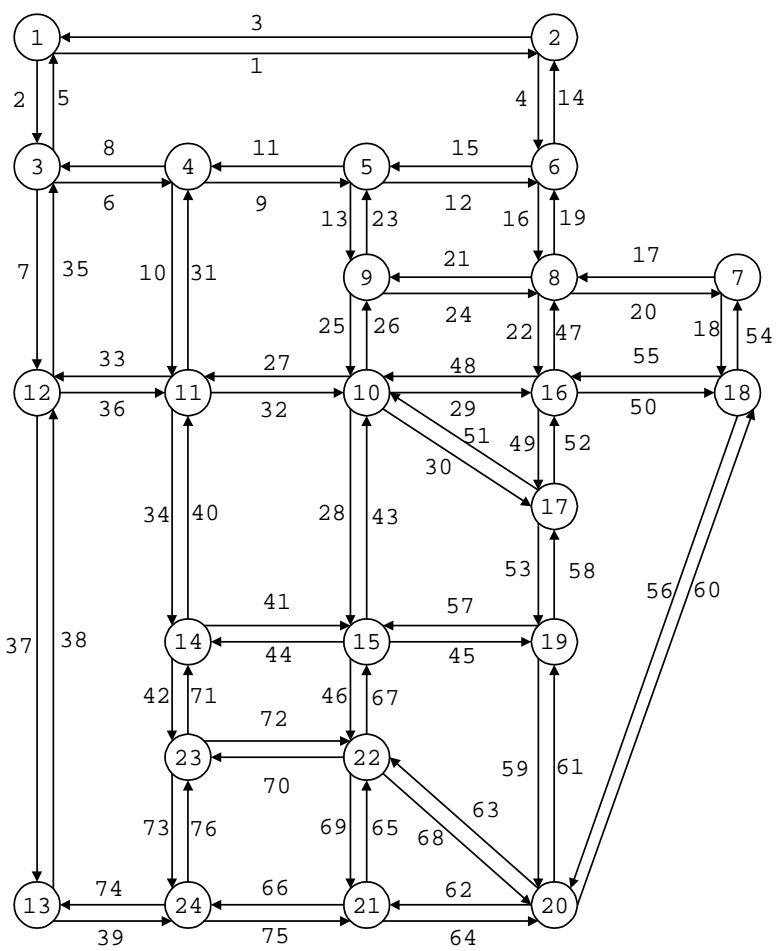

Anaheim Network

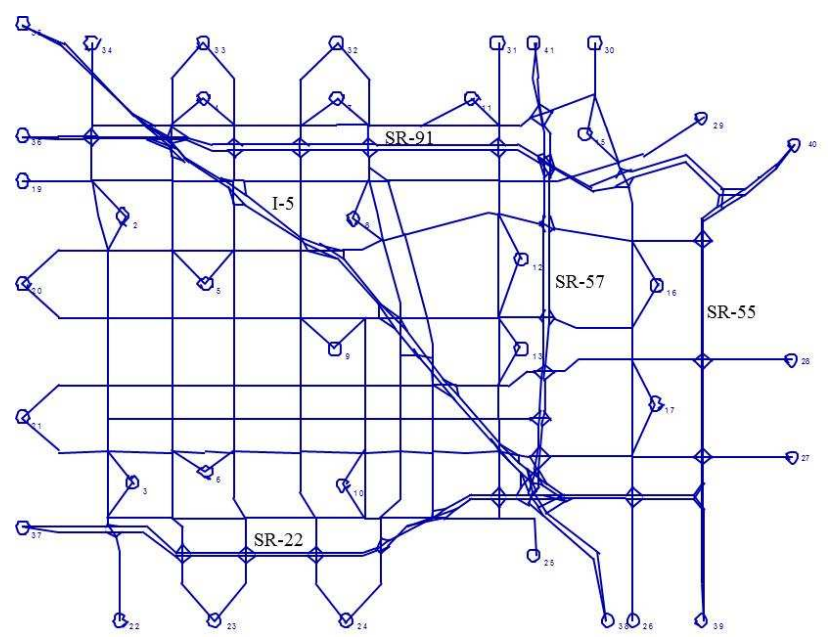

